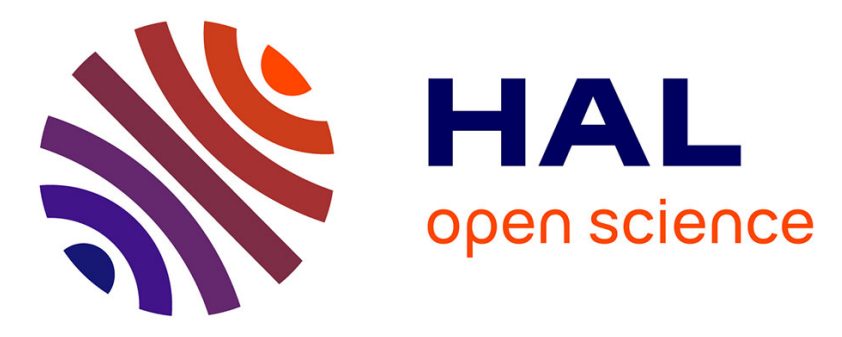

\title{
Modelling plant compensatory effects in plant-insect dynamics
}

\author{
Audrey Lebon, Ludovic Mailleret, Frédéric Grognard, Yves Dumont
}

\section{To cite this version:}

Audrey Lebon, Ludovic Mailleret, Frédéric Grognard, Yves Dumont. Modelling plant compensatory effects in plant-insect dynamics. 4th International Symposium on Plant Growth Modeling, Simulation, Visualization and Applications, (PMA 2012), Institute of Electrical and Electronics Engineers (IEEE). USA., 2012, Shanghai, China. pp.212-219, 10.1109/PMA.2012.6524836 . hal-00848422

\section{HAL Id: hal-00848422 https://hal.inria.fr/hal-00848422}

Submitted on 26 Jul 2013

HAL is a multi-disciplinary open access archive for the deposit and dissemination of scientific research documents, whether they are published or not. The documents may come from teaching and research institutions in France or abroad, or from public or private research centers.
L'archive ouverte pluridisciplinaire HAL, est destinée au dépôt et à la diffusion de documents scientifiques de niveau recherche, publiés ou non, émanant des établissements d'enseignement et de recherche français ou étrangers, des laboratoires publics ou privés. 


\section{Modelling Plant Compensatory Effects in Plant-Insects dynamics}

\author{
Audrey Lebon, Yves Dumont \\ Umr AMAP \\ CIRAD \\ Montpellier, France \\ audrey.lebon@cirad.fr, \\ yves.dumont@cirad.fr
}

\author{
Frédéric Grognard \\ BIOCORE \\ INRIA \\ Sophia-Antipolis, France \\ frederic.grognard@inria.fr
}

\author{
Ludovic Mailleret \\ INRA - CNRS - UNS - UMR ISA - TEAPEA \\ F-06903 Sophia Antipolis \\ INRIA - BIOCORE \\ F-06902 - Sophia Antipolis \\ ludovic.mailleret@sophia.inra.fr
}

\begin{abstract}
Modelling plant-pest interactions is not an obvious task since the involved processes are numerous and complex. We propose a minimal model based on trophic relations and the concept of plant compensation capacity. We only consider three main components in our system: the plant foliar biomass, the compensation capacity, and the pest population. We prove that there exist two threshold parameters, $\mathcal{N}_{1}$ and $\mathcal{N}_{2}$, and show that the system admits different equilibria, which are locally asymptotically stable or unstable, depending on the value of the previous threshold parameters. Finally, we summarize our theoretical results in a bifurcation diagram that allows to discuss possible control strategies to lower the impacts of the pest or even to obtain a better biomass yield.

Index Terms-Mathematical modelling, crop protection, plant-insect interactions, plant compensation, bifurcation diagram
\end{abstract}

\section{INTRODUCTION}

Biological control, i.e. the use of living organisms to control pest invasions, is booming in crop protection. If the part of biological control in the global crop protection market was minimal in $2000(0.2 \%)$, the development of biopesticides and the discovery of new biological control agents has increased the percentage to $2.5 \%$. Biological control programs mainly concern aphids, thrips, lepidopteran and dipteran leaf-chewing or miner caterpillars, mites and scales in almost all agrosystems (forests, greenhouses, fields) [25]. Even if they are currently more and more used, their successes in pest control are mixed and debated in the scientific community. For instance, the establishment frequency of control agents is estimated to be $34 \%-50 \%$ and the total successes for controlling the target population are around 3\% and $11 \%$ for partial controls [15]. In addition, non target effects are a common problem of biological control programs. The damage caused ecologically but also economically can be very important [15]. A well known example is Harmonia axyridis (Pallas), an asian ladybird which proliferates in France but also in North and South America. Initially, this ladybird has been used to control aphids and psyllids and was really efficient. But among other problems, its important proliferation disrupted ecosystems equilibria and led to the extinction of other ladybird species [2], [9], [28].

The relationships between insect populations and plants are complex and their dynamics difficult to predict. They are ruled by a lot of different phenomena such as physiological processes, allelopathic relations, defense mechanisms, trophic relations, etc... Mathematical modelling is a useful tool to understand the dynamics of these biological systems. Models can also agregate knowledge and as such provide a synthetic view on plant-insects interactions.

Most of the time, biological control models only take into account the pest population and its natural enemies (see [17] and references therein) and do not integrate the plant compartment, considering somehow that the crop and the insect populations are not in interaction. In practice, this assumption does not always hold. If the main objective of biological control is to maintain the crop yield above a critical economic threshold, it seems clear that crop growth has to be taken into account in the biological control modelling.

Actually, there exist different plant modelling approaches. Empirical models [23] are the simplest ones. They are directly linked to experimental data and give no biological explanation on the considered phenomena. Process Based Models, in short PBM, developed since the seventies, intend to describe the essential physiological processes in plant growth [27], [16], [11], [3]. Finally, more recent models integrate plant functioning and structure or architecture. These new models are called Functional Structural Plant Models, shortly FSPM [8], [26].

Integrating a plant compartment in a pest-natural 
enemy model entails integrating interactions between plants and insects. This is not an easy task. Indeed, these interactions merge trophic relations, toxins/viruses/phytoplasma injections, resistance and tolerance [24], [1], chemical and mechanistic defenses [12]. Surprisingly, the scientific litterature remains scarce in concrete papers about these interactions and their modelling [10].

Our aim is to model some plant-pest interactions. We built an explanatory model which we were able to study mathematically. We decided not to consider indirect pest or plant effects like toxines, viruses or chemical defenses and to concentrate on the trophic relations and the plant compensation capacity.

Plant compensation is the process by which plants respond positively to recover from the effects of pest injury on plant growth [6]. It is a common phenomenon, which has been repeatedly reported in various plant taxa during the last thirty years. For instance, the Butterprint (Abutilon theophrasti Medik) preserves its growth and reproduction, when, at most, $75 \%$ of defoliation occur (see [22] and references therein); wild radish plants (Raphanus raphanistrum Linné) behaves similarly with only a defoliation up to $25 \%$ [1]. Depending on the authors, plant compensation is assessed through the variation in plant biomass or plant fitness between an undamaged and a damaged plant [21]. If the fitness or biomass of a damaged plant is higher, lower or equal to the fitness or biomass of an undamaged plant, it is termed over- under- or plain compensation respectively. However the "overcompensation" phenomenon is still the matter of a debate [1], [18].

A lot of processes have been proposed to explain compensation [22], [18] :

- carbon reallocation.

- up-regulation of the primary metabolism (increased photosynthesis rate, increased growth rate, increased fruit set percentage)

- release of buds and meristems dormancy (increased branching)

The compensation capacity mainly leans on the carbohydrate source-sink dynamics. Mature photosynthesis leaves and stored reserves are sources. All other parts of the plants and the storage compartment can be considered as sinks, while new leaves, flowers and fruits are particulary strong sinks. Sink strength can regulate the photosynthesis of leaves. If there exists a competition (several sinks) for one given source, the strongest sinks will receive a greater part of resources [14], [5]. A pest attack increases the relative sink strength because of the leaves biomass consumption. The total carbon assimilation should indeed decrease if source leaves are removed. However, the increased carbon demand on the remaining leaves can then potentially trigger an increase of the photosynthesis rate, a faster carbon fixation and translocation than in undamaged plants [22]. The plant can thus compensate the pest attack. This photosynthesis increase has been experimentally described using non destructive and non invasive chlorophyll fluorescence measurements [22].

The compensation mechanisms can also simply be triggered by a pest elicited increase of the primary metabolism [18] : the presence and action of the pest is detected by chemical receptors that lead to the activation of the compensation mechanisms. This hypothesis is supported by the study of pest larvae regurgitants like, for instance, the Guatemalan potato moth (Tecia solanivora (Povolny)) [18].

The timing of pest attacks also seems to have its importance on the intensity of the compensation response. Indeed, a pre-flowering attack on lebanese cucumber (Cucumis sativus, Linné) has a positive effect on vegetative biomass and fruit production whereas during flowering, the damaged plants can only compensate in terms of vegetative biomass and not in fruit production [22].

Compensation capacity can be considered as an evolution of plants which were not able to compensate but grew with high level of herbivory [13], [22]. In pestfree conditions, the plants that cannot compensate have a better growth and dominate the environment. However, if a pest attack occurs, they will be more affected than the compensating ones [13], [22]. It means that in an non-limited growth environment with a constantly high pest pressure, the compensating plants will be favoured.

Our paper is organized as follows. In Section II, we introduce the model. Our approach tries to be as general as possible and the model is studied without concentrating on a particular biological situation. In section III, we discuss the different theoretical results. The paper ends with a brief conclusion.

\section{MODEL DESCRIPTION}

Our model consists of three coupled ordinary differential equations, taking into account the plant foliar biomass, $B$, a variable related to the plant compensation, $V$, and the pest population, $R$.

Our model is built around the following hypotheses :

- For the plant compartment :

- non-restricting growth conditions, i.e. greenhouses growth conditions, implies no abiotic stresses and, no competition for resources, 
- crop representation is reduced to its foliar biomass, simplifying hypothesis whose validity depends on the considered pest,

- no chemical or mechanistic defenses but compensation capacity in order to simplify the plant-insect interactions

- all fixed carbon is used for vegetative growth and compensation capacity

- For the pest compartment :

- one undifferentiated population of larvae,

- impact on plant's growth rate and on the foliar biomass (leaf-chewing or leaf-miner caterpillars),

- no cannibalism,

- the insect birth rate depends on the plant biomass.

The plant net growth results from three main processes: gross primary growth, compensation capacity and maintenance process. We denote by $r_{B}$ the biomass growth rate and $V$ the variable representing the plant compensation capacity. Initially, $V$ is neutral, i.e. equal to 1 : the plant grows according to its biomass gross growth rate discounted by the maintenance $\delta B^{2}$, modelled as an accelerating (convex) function of biomass. However, the compensation capacity can increase according to $\beta(R)$ which is the plant compensation response to a pest invasion, varying among the plant species. We assume that the plant compensation capacity is proportional to the pest population, i.e. $\beta(R)=\beta R$ with $\beta \geq 0$. This assumption is the boundary case of a concave, increasing and monotonic function that could be chosen for $\beta(R)$. When a pest attack occurs, the compensation capacity increases and so does the plant gross primary growth. When the pest disappears, the compensation capacity $V$ goes back to its initial level according to a restoring force $a$. However, in the literature, even if the compensation response is clearly linked to the level of pest attack, there is no consensus on the real impact of pest density on the plant response. For instance, in the case of the Guatemalan potato moth (Tecia solanivora), below $10 \%$ of damage, the potato yield increases, otherwise the benefit of the pest attack decreases [18]. In contrary, for lebanese cucumber, $40 \%$ or, even, $80 \%$ of damage caused by brown garden snails (Helix aspersa Müller), have an equivalent (positive) effect on the compensation capacity [22].

We consider that the population dynamics are determined by two main parameters: $\alpha$ and $\mu$, that represent, respectively, the pest net growth rate per unit of consumed biomass and, the pest mortality rate. Finally, we assume that the pest population impacts plant growth at different levels: the gross growth rate $r_{B}$ becomes $\frac{r_{B}}{\gamma R+1}$, i.e. the gross growth rate decreases with the pest attack intensity. Moreover an additional term is considered to take into account the pest biomass consumption through a mass action principle, i.e. $-\phi B R$, where $\phi$ is the specific biomass consumption rate.

Altogether, we obtain the following system :

$$
\left\{\begin{array}{l}
\dot{B}=\frac{r_{B} B V}{\gamma R+1}-\delta B^{2}-\phi B R \\
\dot{V}=a(1-V)+\beta R \\
\dot{R}=\alpha B R-\mu R .
\end{array}\right.
$$

with the following positive initial conditions :

$$
\left\{\begin{array}{l}
B(0)=B_{0}, \\
V(0)=1 \\
R(0)=R_{0} .
\end{array}\right.
$$

If there is no pest attack, the crop biomass grows normally according to its biomass growth rate. In that case, we assume, for sake of simplicity, that the plant biomass follows a logistic growth. When an attack occurs, there are different possibilities according to the compensation capacity (extinction of the pest population or coexistence of the two compartments).

In the case where plants cannot compensate pest attacks, i.e. $\beta=0$, we can assume $V$ is always equal to 1 , its neutral value and the system reduces to:

$$
\left\{\begin{array}{l}
\dot{B}=\frac{r_{B} B}{\gamma R+1}-\delta B^{2}-\phi B R \\
\dot{R}=\alpha B R-\mu R .
\end{array}\right.
$$

\section{DYNAMICS OF THE MODEL}

The solution of system (1) is well-defined and stays in the non-negative orthant $\mathbb{R}_{+}^{3}$ so that system (1) is biologically well-posed.

\section{A. Existence of equilibria}

Looking for the equilibria in system (1) is equivalent to solve the following system:

$$
\left\{\begin{array}{l}
0=B^{*}\left(\frac{r_{B} V^{*}}{\gamma R^{*}+1}-\delta B^{*}-\phi R^{*}\right), \\
0=a\left(1-V^{*}\right)+\beta R^{*} \\
0=R^{*}\left(\alpha B^{*}-\mu\right) .
\end{array}\right.
$$

We identify two trivial pest-free equilibria: $(0,1,0)$ and $\left(\frac{r_{B}}{\delta}, 1,0\right)$. Assuming $R^{*}>0$ leads to:

$$
V^{*}=1+\frac{\beta R^{*}}{a}
$$

and

$$
B^{*}=\frac{\mu}{\alpha}
$$


which introduced into equation $(4)_{1}$ give :

$$
-\phi \gamma\left(R^{*}\right)^{2}+\left(\frac{r_{B} \beta}{a}-\phi-\delta B^{*} \gamma\right) R^{*}+r_{B}-\delta B^{*}=0 .
$$

Let us consider the following threshold parameters

$$
\begin{gathered}
\mathcal{N}_{1}=\frac{\beta r_{B}}{a\left(\phi+\gamma r_{B}\right)}, \\
\mathcal{N}_{2}=\frac{\mu \delta}{r_{B} \alpha} .
\end{gathered}
$$

$\mathcal{N}_{1}$ represents the net plant response to the pest attack, as determined from the increase in gross growth rate linked to plant compensation and the co-occurent direct decrease due to plant consumption by the pest; and $\frac{1}{\mathcal{N}_{2}}$ is the reproductive number of the pest at biomass equilibrium.

Re-writting (5) with the threshold parameters :

$$
b R^{* 2}+c R^{*}+d=0
$$

with :

$$
\begin{gathered}
b=\phi \gamma, \\
c=\left(\phi+\gamma r_{B}\right)\left(1-\mathcal{N}_{1}\right)+\gamma r_{B}\left(\mathcal{N}_{2}-1\right), \\
d=r_{B}\left(\mathcal{N}_{2}-1\right) .
\end{gathered}
$$

The two solutions of (6) are given by

$$
\left\{\begin{array}{l}
R_{1}^{*}=\frac{-c-\sqrt{\Delta}}{2 b} \\
R_{2}^{*}=\frac{-c+\sqrt{\Delta}}{2 b}
\end{array}\right.
$$

with $\Delta=c^{2}-4 b d$ and $R_{2}^{*} \geq R_{1}^{*}$ if both roots are real (we write $R_{1,2}^{*}$ if they are identical). Since $R_{1}^{*} R_{2}^{*}=\frac{d}{b}$ and since $b>0$, both roots are real and of opposite sign if and only if $d<0$, that is if $\mathcal{N}_{2}<1$; in that case $R_{2}^{*}>0$ and $R_{1}^{*}<0$. Now, if $d>0$, both roots are either real and of the same sign or complex conjugate; they then only exist in our domain of definition if they are both real and positive. This is achieved if their sum $R_{1}^{*}+R_{2}^{*}=-\frac{c}{b}>0$ (that is if $c<0$ ) and $\Delta>0$. The latter writes $c^{2}>4 b d$ which, since $c<0$ becomes $-c>2 \sqrt{b d}$. We conclude that (6) presents two positive roots if and only if $\mathcal{N}_{2}>1$ and

$$
\mathcal{N}_{1}>1+f\left(\mathcal{N}_{2}\right)
$$

with $f\left(\mathcal{N}_{2}\right)=\frac{2 \sqrt{\gamma \phi r_{B}\left(\mathcal{N}_{2}-1\right)}+\gamma r_{B}\left(\mathcal{N}_{2}-1\right)}{\phi+\gamma r_{B}}$, the $c<0$ condition being also encompassed in the latter one. In all other cases, it has no positive root.

The results are summarized in Table I on page 5 . Note that case (iv) to (vi) (Table I) are only realised for precise values of the parameters, so that they will not be encountered in nature. Yet they define curves in the
$\left(\mathcal{N}_{1}, \mathcal{N}_{2}\right)$ plane at which bifurcations occur: these curves separates the $\left(\mathcal{N}_{1}, \mathcal{N}_{2}\right)$ plane into regions in which the model has different qualitative behaviors corresponding to cases (i) to (iii).

Remark 1. When $\mathcal{N}_{2}>1, B^{*}=\frac{\mu}{\alpha}>\frac{r_{B}}{\delta}$, i.e. in case (iii), the final plant-pest co-existence biommass is higher than the pest-free biomass. The positive equilibria in section (iii) are overcompensation cases. At the contrary, the positive biomass equilibrium in case (i) is lower than the pest-free biomass and thus, is an under-compensation case.

\section{B. Local asymptotic stability/instability of the equilibria}

Following [4], the local asymptotic stability or instability of each equilibrium is studied by computing the eigenvalues of the Jacobian Matrix $\mathcal{J}(X)$ related to system (1), that is $\mathcal{J}(X)=$

$$
\left(\begin{array}{ccc}
\frac{r_{B} V}{\gamma R+1}-2 \delta B-\phi R & \frac{r_{B} B}{\gamma R+1} & \frac{-\gamma r_{B} B V}{(\gamma R+1)^{2}}-\phi B \\
0 & -a & \beta \\
\alpha R & 0 & \alpha B-\mu
\end{array}\right) .
$$

- When $X^{*}=(0,1,0)$, we have

$$
\mathcal{J}\left(X^{*}\right)=\left(\begin{array}{ccc}
r_{B} & 0 & 0 \\
0 & -a & \beta \\
0 & 0 & -\mu
\end{array}\right)
$$

which implies that the equilibrium is always unstable, because it has one positive eigenvalue, $r_{B}$. $(0,1,0)$ is a saddle point.

- When $X^{*}=\left(\frac{r_{B}}{\delta}, 1,0\right)$, we have

$$
\mathcal{J}\left(X^{*}\right)=\left(\begin{array}{ccc}
-r_{B} & \frac{r_{B}^{2}}{\delta} & -\frac{\gamma r_{B}^{2}}{\delta}-\frac{\phi r_{B}}{\delta} \\
0 & -a & \beta \\
0 & 0 & \frac{\alpha r_{B}}{\delta}-\mu
\end{array}\right)
$$

which implies that the equilibrium is locally asympotically stable if $\mathcal{N}_{2}>1$. If $\mathcal{N}_{2}<1$, the equilibrium is unstable: it is a saddle point.

- When $X^{*}=\left(B^{*}, V^{*}, R^{*}\right)$, with $R^{*}=R_{1}^{*}$ or $R_{2}^{*}$ or $R_{1,2}^{*}$, we use the previous computations :

$$
\left\{\begin{array}{l}
B^{*}=\frac{\mu}{\alpha} \\
0=B^{*}\left(\frac{r_{B} V^{*}}{\gamma R^{*}+1}-\delta B^{2 *}-\phi R^{*}\right) .
\end{array}\right.
$$

With this second equation, we can write :

$$
\begin{aligned}
& -\phi R^{*}=\delta B^{*}-\frac{r_{B} V^{*}}{\gamma R^{*}+1}, \\
& r_{B} V^{*} B^{*}=\left(\phi B^{*} R^{*}+\delta B^{* 2}\right)\left(\gamma R^{*}+1\right) .
\end{aligned}
$$


Table I

TABLE OF THE DIFFERENT CASES FOR THE POSITIVE EQUILIBRIA

\begin{tabular}{|c|c|c|c|}
\hline Cases & $\mathcal{N}_{1}$ conditions & $\mathcal{N}_{2}$ conditions & Equilibria \\
\hline i & $\mathcal{N}_{1} \geq 0$ & $0 \leq \mathcal{N}_{2}<1$ & $\begin{array}{c}\text { one positive equilibrium } \\
\left(B^{*}, V_{2}^{*}, R_{2}^{*}\right)\end{array}$ \\
\hline ii & $0<\mathcal{N}_{1}<1+f\left(\mathcal{N}_{2}\right)$ & $\mathcal{N}_{2}>1$ & no positive equilibrium \\
\hline iii & $\mathcal{N}_{1}>1+f\left(\mathcal{N}_{2}\right)$ & $\mathcal{N}_{2}>1$ & $\begin{array}{c}\text { two positive equilibria } \\
\left(B^{*}, V_{1}^{*}, R_{1}^{*}\right),\left(B^{*}, V_{2}^{*}, R_{2}^{*}\right)\end{array}$ \\
\hline iv & $0<\mathcal{N}_{1} \leq 1$ & $\mathcal{N}_{2}=1$ & no positive equilibrium \\
\hline v & $\mathcal{N}_{1}>1$ & $\mathcal{N}_{2}=1$ & $\begin{array}{c}\text { one positive equilibrium } \\
\left(B^{*}, V_{2}^{*}, R_{2}^{*}\right)\end{array}$ \\
\hline vi & $\mathcal{N}_{1}=1+f\left(\mathcal{N}_{2}\right)$ & $\mathcal{N}_{2}>1$ & $\begin{array}{c}\text { one positive equilibrium } \\
\left(B^{*}, V^{*}, R_{1,2}^{*}\right)\end{array}$ \\
\hline
\end{tabular}

Putting $(7)_{1}$ into $\mathcal{J}_{11}\left(X^{*}\right)$ and $(7)_{2}$ into $\mathcal{J}_{13}\left(X^{*}\right)$, we have the following useful relations between $B^{*}, V^{*}, R^{*}$,

$$
\left\{\begin{array}{l}
B^{*}=\frac{\mu}{\alpha} \\
\frac{r_{B} V}{\gamma R+1}-2 \delta B-\phi R=-\delta B^{*}, \\
\frac{-\gamma r_{B} B^{*} V^{*}}{\left(\gamma R^{*}+1\right)^{2}}-\phi B^{*}=\frac{-\gamma\left(\phi B^{*} R^{*}+\delta B^{* 2}\right)}{\left(\gamma R^{*}+1\right)}-\phi B^{*},
\end{array}\right.
$$

which imply the following simplifications in the Jacobian Matrix, that in $\mathcal{J}\left(X^{*}\right)$ :

$$
\left(\begin{array}{ccc}
-\delta B^{*} & \frac{r_{B} B^{*}}{\gamma R^{*}+1} & \frac{-\gamma\left(\phi B^{*} R^{*}+\delta B^{* 2}\right)}{\left(\gamma R^{*}+1\right)}-\phi B^{*} \\
0 & -a & \beta \\
\alpha R^{*} & 0 & 0
\end{array}\right) .
$$

We then calculate the characteristic polynomial :

$$
\begin{gathered}
P(\lambda)=\operatorname{det}\left(\mathcal{J}\left(X^{*}\right)-\lambda I d\right) \\
=\left|\begin{array}{ccc}
-\delta B^{*}-\lambda & \frac{r_{B} B^{*}}{\gamma R^{*}+1} & \frac{-\gamma\left(\phi B^{*} R^{*}+\delta B^{* 2}\right)}{\left(\gamma R^{*}+1\right)}-\phi B^{*} \\
0 & -a-\lambda & \beta \\
\alpha R^{*} & 0 & -\lambda
\end{array}\right| .
\end{gathered}
$$

Expanding $P(\lambda)$ gives:

$$
\begin{aligned}
P(\lambda)= & -\delta a B^{*} \lambda-\delta B^{*} \lambda^{2}-a \lambda^{2}-\lambda^{3} \\
& +\frac{\alpha r_{B} B^{*} \beta R^{*}}{\gamma R^{*}+1}-\frac{\alpha R^{* 2} \gamma a \phi B^{*}}{\gamma R^{*}+1} \\
& -\frac{\alpha R^{*} \delta a B^{* 2}}{\gamma R^{*}+1}-\alpha R^{*} \phi a B^{*}-\frac{\alpha R^{* 2} \gamma \phi B^{*} \lambda}{\gamma R^{*}+1} \\
& -\frac{\alpha R^{*} \delta \gamma B^{* 2} \lambda}{\gamma R^{*}+1}-\alpha R^{*} \phi B^{*} \lambda .
\end{aligned}
$$

Then, rearranging the terms, the characteristic polynomial becomes $P(\lambda)$ :

$$
\begin{aligned}
& =-\lambda^{3}-\lambda^{2}\left(-\delta B^{*}-a\right) \\
& -\lambda\left(-\delta a B^{*}-\frac{\alpha R^{* 2} \gamma \phi B^{*}}{\gamma R^{*}+1}-\frac{\alpha R^{*} \delta \gamma B^{* 2}}{\gamma R^{*}+1}-\alpha R^{*} B^{*} \phi\right) \\
& +\frac{\alpha r_{B} B^{*} \beta R^{*}}{\gamma R^{*}+1}-\frac{\alpha R^{* 2} \gamma a \phi B^{*}}{\gamma R^{*}+1}-\frac{\alpha R^{*} \delta a \gamma B^{* 2}}{\gamma R^{*}+1} \\
& -\alpha R^{*} \phi a B^{*}
\end{aligned}
$$

To show the local asymptotic stability of a positive equilibrium $\left(B^{*}, V^{*}, R^{*}\right)$, we need to show that all roots of $P(\lambda)$ have negative real part. To prove it, we use the Routh-Hurwitz criterion [7]. After some tedious but straightforward computations, we deduce that $\left(B^{*}, V^{*}, R^{*}\right)$ is locally asymptotically stable if $R^{*}>\frac{-c}{2 b}$, i.e $R^{*}>$ $\frac{-\left(\phi+\gamma r_{B}\right)\left(1-\mathcal{N}_{1}\right)+\gamma r_{B}\left(\mathcal{N}_{2}-1\right)}{2 \phi \gamma}$ which is always the case for $\left(B^{*}, V_{2}^{*}, R_{2}^{*}\right)$ and never for $\left(B^{*}, V_{1}^{*}, R_{1}^{*}\right)$.

We summarize the previous results in the following theorem

\section{Theorem 1. .}

- The equilibria $(0,1,0)$ and $\left(B^{*}, V_{1}^{*}, R_{1}^{*}\right)$ (when it exists) are always unstable.

- $\left(\frac{r_{B}}{\delta}, 1,0\right)$ is locally asymptotically stable if $\mathcal{N}_{2}>1$ and unstable if $\mathcal{N}_{2}<1$.

- $\left(B^{*}, V_{2}^{*}, R_{2}^{*}\right)$ (when it exists) is always locally asymptotically stable.

Remark 2. The cases (iv), (v) and (vi) lead to nonhyperbolic equilibria.

We summarize the previous results in Fig. 1.

Fig. 1 shows the different equilibria of system (1) and their stability but not its backward bifurcation when $N_{1}>1$. A backward bifurcation is a particular transcritical bifurcation : one of the three biologically feasible branches of the transcritical bifurcation in the neighborhood of $\frac{1}{\mathcal{N}_{2}}=1$ is locally stable, here $\left(B^{*}, V_{2}^{*}, R_{2}^{*}\right)$. Because the remaining two biologically feasible branches (corresponding to non-negative population states, $\left(\frac{r_{B}}{\delta}, 1,0\right)$ and $\left.\left(B^{*}, V_{1}^{*}, R_{1}^{*}\right)\right)$ are locally unstable, variations in the basic reproductive ratio lead to discontinuous changes in the asymptotic dynamics of the system [19]. Such a bifurcation thus modifies what is generally expected, which is: when the reproductive number, $\frac{1}{\mathcal{N}_{2}}$, is lower than 1 , the pest populations goes 


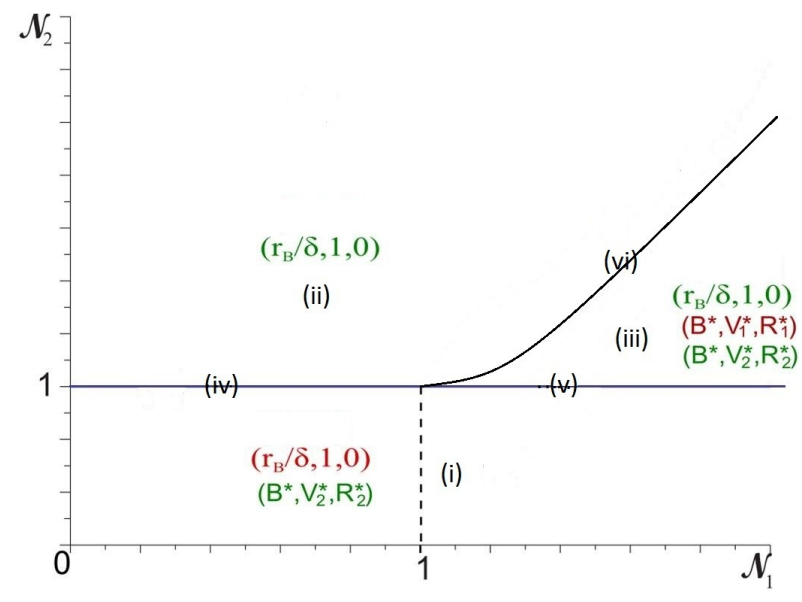

Figure 1. Bifurcation diagram summarizing different situations. The green equilibria are stable and the red ones are unstable. $(0,1,0)$ is not illustrated since it always unstable. The numbers correspond to the different cases of Table 1 .

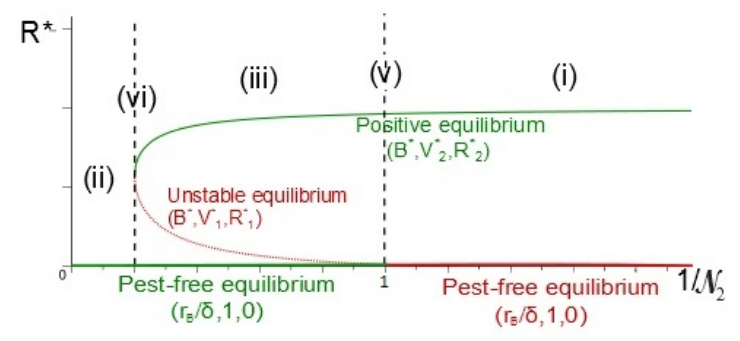

Figure 2. Backward bifurcation of system 1. The green equilibria are stable and the red one is unstable. The numbers correspond to the different cases of Table 1 .

extinct while it can otherwise persist. In the case of a backward bifurcation (Fig. 2), in the same situation, small invasions still cannot succeed (the pest-free equilibrium exists), but there exists a positive equilibrium where pests are present. The system will converge to the pest-free equilibrium or the positive one according to the initial pest invasion, all others parameters being fixed.

\section{Discussion}

\section{A. Model dynamics}

The bifurcation diagram (Fig. 1) summarizes many important informations regarding system (1) dynamics. It merges different equilibria situations, numbered from (i) to (vi).

In situation (i) the compensation capacity allows the maintenance of the pest population, $R_{2}^{*}$, with a biomass $B^{*}$ lower than the pest free biomass. Thus, system (1) converges to the positive equilibrium $\left(B^{*}, V_{2}^{*}, R_{2}^{*}\right)$.
Situation (i) may represent cases of no-compensation and under-compensation.

In situation (ii), contrarily to situation (i), the compensation capacity, $V$, does not allow the maintenance of the pest population, and the plant will reach its normal amount of biomass. System (1) will converge to equilibrium $\left(\frac{r_{B}}{\delta}, 1,0\right)$.

Situation (iii) is particular. Indeed, depending on the initial pest population, the plant compensatory response, which is proportional to the pest population, i.e. $\beta(R)=\beta R$, drives system (1) to one of the LAS equilibria, $\left(\frac{r_{B}}{\delta}, 1,0\right)$ or $\left(B^{*}, V_{2}^{*}, R_{2}^{*}\right)$. With $(\mathrm{B}, \mathrm{V})$ initially at $\left(\frac{r_{B}}{\delta}, 1\right)$, if the initial pest population is small, the compensatory response is weak, so that the biomass only slowly increases from $\frac{r_{B}}{\delta}$, while $R$ quickly goes back to zero because $\frac{\alpha r_{B}}{\delta}-\mu<0$, anihilating the compensation effect; hence $B$ settles to $\frac{r_{B}}{\delta}$ again. In this situation, the plant compensation thus acts as a mechanism generating an Allee effect in the pest population. In the second case, when the pest population is sufficiently large, the biomass reaches its equilibrium before the pest disappears. This positive equilibrium shows a final biomass higher than in the other case, and thus can be caracterised as an overcompensation one, as said in Remark 1.

Cases (iv), (v) and (vi) determine the borderlines between the previous generic cases, where bifurcations take place; their types can easily be identified on Fig. 1. When transition from region (i) to region (iii) through curve (iv) takes place, there is an exchange of stability between $\left(\frac{r_{B}}{\delta}, 1,0\right)$ and $\left(B^{*}, V_{1}^{*}, R_{1}^{*}\right)$ which collide; this corresponds to a transcritical bifurcation. Similarly, on curve (v), a transcritical bifurcation takes place between $\left(\frac{r_{B}}{\delta}, 1,0\right)$ and $\left(B^{*}, V_{2}^{*}, R_{2}^{*}\right)$. Finally curve (vi) corresponds to a saddle node bifurcation: unstable $\left(B^{*}, V_{1}^{*}, R_{1}^{*}\right)$ and stable $\left(B^{*}, V_{2}^{*}, R_{2}^{*}\right)$ collide and disappear: they become complex. The case where $\beta=0$ is represented when $\mathcal{N}_{1}=0$ with the same equilibria and stability as in cases (i) and (ii).

\section{B. How to choose the best control strategy?}

The bifurcation diagram (Fig. 1) gives also some potential control of system (1). Indeed, the most interesting equilibrium is the overcompensation one, i.e the positive equilibrium $\left(B^{*}, V_{2}^{*}, R_{2}^{*}\right)$ in case (iii). It is thus desirable that model (1) reaches this equilibrium. The bifurcation diagram shows also that switching from one equilibrium to another is possible through the threshold parameters $\mathcal{N}_{1}$ and $\mathcal{N}_{2}$.

To switch from case (i) to case (iii), the system has to be in the area where $\mathcal{N}_{1}>1$, and the control should impose an increase of $\mathcal{N}_{2}=\frac{\mu \delta}{\alpha r_{B}}$ (Fig. 3). To do that, 


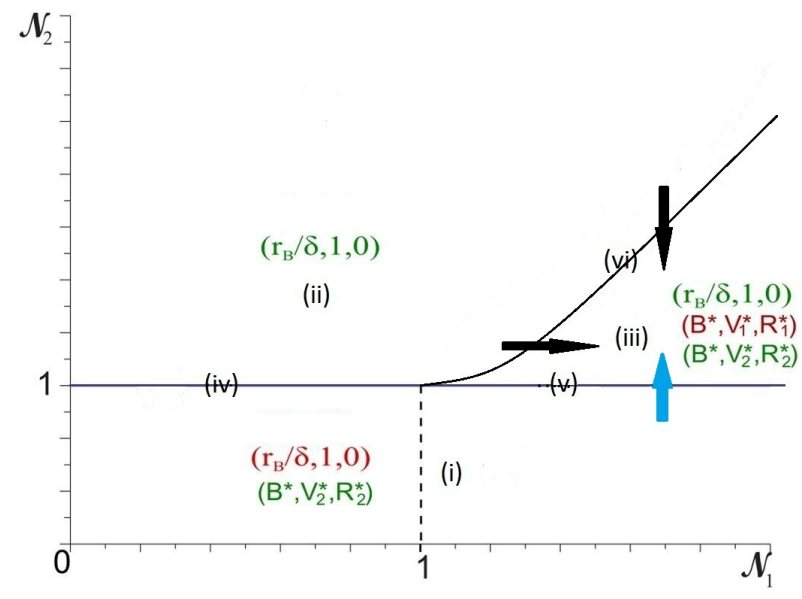

Figure 3. Bifurcation diagram with the different possible controls. The blue arrow shows the switching from case i to case iii and the dark ones the switching from case ii to case iii. The numbers and the equilibria are the same than in Fig.1

there are three different possibilities : increase the pest mortality rate, $\mu$, decrease the pest net growth rate, $\alpha$ or increase $\frac{\delta}{r_{B}}$. However, increasing $\frac{\delta}{r_{B}}$ is of no interest since it would bring the parameters from region (i) to (iii) without changing the final plant-pest co-existence biomass $B^{*}=\frac{\mu}{\alpha}$ which does not depend on $\frac{\delta}{r_{B}}$; even worst: this could bring the solution to $\left(\frac{r_{B}}{\delta}, 1,0\right)$ with a pest-free biomass level $\frac{r_{B}}{\delta}$ smaller than the plant-pest co-existence biomass, $B^{*}=\frac{\mu}{\alpha}$, originally achieved in region (i). On the contrary, changing $\mu$ or $\alpha$ can increase the plant-pest co-existence biomass level at equilibrium and this can be done with pest control means: Bacillus thuringiensis as a poison [20], moulting hormone, eggs poison, etc... . As explained in the previous subsection, the initial pest invasion must nevertheless be high enough to switch to the positive equilibrium of case (iii) and not $\left(\frac{r_{B}}{\delta}, 1,0\right)$. However, even if the solution eventually converges to $\left(\frac{r_{B}}{\delta}, 1,0\right)$ this would still yield a larger pest-free biomass level than the original plant-pest coexistence level, $B^{*}=\frac{\mu}{\alpha}$, achieved by equilibrium $\left(B^{*}, V_{2}^{*}, R_{2}^{*}\right)$ in region (i).

If the system is in the area where $\mathcal{N}_{1}<1$, the only possible improvement is to switch from case (i) to case (ii) by increasing $\mathcal{N}_{2}$. This control should be done if $B^{*}<<\frac{r_{B}}{\delta}$ to have a worthy final biomass difference. This is in fact the only action that can be taken when the system does not have any compensation capacity, i.e. $\mathcal{N}_{1}=0$. By manipulating the pest parameters, i.e. $\mu$ and/or $\alpha$, we can force pest exclusion and guarantee the biomass to converge to its pest-free level.

The case of switching from case (ii) to case (iii) is particular. When the initial pest population is low, situations (ii) and (iii) are similar: the system may converge to the same equilibrium, $\left(\frac{r_{B}}{\delta}, 1,0\right)$, at least locally. However, in the case of an important initial pest infestation, a control can be proposed. There are indeed, two different possibilities (Fig. 3). If the system is in the area where $\mathcal{N}_{1}<1$, switching from case (ii) to case (iii) means increasing $\mathcal{N}_{1}$. This is possible by increasing $\beta$ or decreasing $a$, i.e. selecting a plant with a more important and efficient compensation capacity, or by reducing the pest impact on the plant by decreasing $\phi$ or $\gamma$, i.e. changing the plant attraction to pest or the pest metabolism. However, decreasing $\phi$ or $\gamma$ changes the curve (vi) and thus modifies section (iii). It means that decreasing these parameters would possibly not bring system (1) to the overcompensation equilibrium, $\left(B^{*}, V_{2}^{*}, R_{2}^{*}\right)$, and even if in section (iii).

If the system is in the area where $\mathcal{N}_{1}>1$, it is possible to switch from case (ii) to case (iii) by decreasing $\mathcal{N}_{2}$. This is equivalent to increase the pest net growth rate, $\alpha$, and/or to decrease the pest mortality rate, $\mu$. This latter result seems to be counterintuitive but is in accordance with the assumptions made on plant compensation: "pest favors plant growth". Indeed, by decreasing $\mu$ or increasing $\alpha$, the pest population grows and with it, the plant compensatory response which allows the pest installation with a higher biomass than previously. That way, overcompensation helps to increase the final biomass.

\section{CONCLUSION}

This study focused on trophic relations which are basic interactions between a plant and an insect population. We introduced a compensation capacity which is a first step in plant tolerance to handle pest attack. Despite a relative simplicity, our model provides different results that seem interesting for a plant-insect system and its control. In particular, our study highlights two threshold parameters, $\mathcal{N}_{1}$ and $\mathcal{N}_{2}$, that aggregate all the model parameters of respectively biomass and pest population. Moreover, they characterize the dynamics of the system and give simple indications on the plant or insect parameters that may be modified to choose appropriate and efficient control strategies. In this case, mathematical modelling can really be complementary to the experiments. It can indeed highlight emerging properties, ask new questions that could be useful to build future experiments and, help to design control strategies.

\section{REFERENCES}

[1] A. A. Agrawal. Overcompensation of plants in response to herbivory and the by-product benefits of mutualism. Trends in plant science, 5(7):309-313, Juillet 2000. 
[2] G. W. Angalet, J. M. Tropp, and A. N. Eggert. Coccinella septempunctata in the united states: Recolonizations and notes on its ecology. Environmental Entomology, 8(5):896-901, 1979.

[3] N Brisson, B Mary, D Ripoche, MH Jeuffroy, F Ruget, B Nicoullaud, P Gate, F Devienne-Barret, R Antonioletti, C Durr, G Richard, N Beaudoin, S Recous, X Tayot, D Plenet, P Cellier, JM Machet, JM Meynard, and R Delecolle. Stics: a generic model for the simulation of crops and their water and nitrogen balances. i. theory and parameterization applied to wheat and corn. Agronomie, 18(5-6):311-346, 1998.

[4] E. Coddington and N. Levinson. Theory of Ordinary Differential Equations. McGraw-Hill, New York, 1955.

[5] M. Dingkuhn, D. Luquet, A. Clément-Vidal, L. Tambour, H.K. Kim, and Y.H. Song. Scale and Complexity in Plant Systems Research: Gene-Plant-Crop Relations, chapter Chapter 13 : Is Plant Growth driven by Sink Regulation ? Implications for crop models, phenotyping approaches and ideotypes. Springer, 2007.

[6] Rubia-Sanchez E., Suzuki Y., Miyamoto K., and Watanabe T. The potential for compensation of the effects of the brown planthopper nilaparvata lugens stal (homoptera: Delphacidae) feeding on rice. Crop Protection, 18(1):39-45, 1999.

[7] F. R. Gantmacher. Applications of the theory of matrices. Interscience Publishers, Ltd., 1959.

[8] C. Godin and H. Sinoquet. Functional-structural plant modelling. New Physiologist, 166(3):705, 2005.

[9] D.J. Greathead. Benefits and risks of classical biological control. Cambridge University Press, Plant and Microbial Biotechnology Research, (4):53-63, 1995.

[10] I. Grechi, N. Hilgertc, B. Sauphanora, R. Senoussid, and F. Lescourreta. Modelling coupled peach tree-aphid population dynamics and their control by winter pruning and nitrogen fertilization. Ecological modelling, (221):2363-2373, 2010.

[11] E. Heuvelink. Evaluation of a dynamic simulation model for tomato crop growth and development. Annals of Botany, (83):413-422, 1999.

[12] K. Ito and S. Sakai. Optimal defense strategy against herbivory in plants : Conditions selecting for induced defense,constitutive defense, and no-defense. Journal of Theoretical Biology, (260):453-459, 2009.

[13] J. Järemo and E. Palmqvist. Plant-compensatory growth : a conquering strategy in plant-herbivore interactions? Evolutionary Ecology, 15:91-102, 2001.

[14] P. Kaitaniemie and T. Honkanen. Simulating source-sink control of carbon and nutrient translocation in a modular plant. Ecological modelling, 88:227-240, 1996.

[15] S.M. Louda, R.W. Pemberton, M.T. Johnson, and P.A. Follett. Nontarget effects-the achilles' heel of biological control? retrospective analyses to reduce risk associated with biocontrol introductions. Annual Review of Entomology, 48:365-396, 2003.

[16] L.F.M Marcelis, E. Heuvelink, and J. Goudriaan. Modelling biomass production and yield of horticultural crops: a review. Scientia Horticulturae, 74(1-2):83 - 111”, 1998.

[17] S. Nundloll. Dos and Don'ts in augmentative biological control : insights from mathematical modelling. $\mathrm{PhD}$ thesis, Université de Nice-Sophia Antipolis, 2010.

[18] K. Poveda, M.I Gomès Jimènez, and A. Kessler. The enemy as ally: herbivore-induced increase in crop yield. Ecological Applications, 20(7):1787-1793, 2012.

[19] T.C Reluga, J. Medlock, and A.S. Perelson. Backward bifurcations and multiple equilibria in epidemic models with structured immunity. Journal of Theoretical Biology, 252:155-165, 2008.

[20] Vincent Sanchis. From microbial sprays to insect-resistant transgenic plants: history of the biospesticide Bacillus thuringiensis. a review. Agronomy for sustainable development, 31(1):217-231, 2011.

[21] S.Y. Strauss and A.A. Agrawal. The ecology and evolution of plant tolerance to herbivory. Trends in ecology evolution, 14:179-185, 199.
[22] V.P. Thomson, S.A. Cunningham, M.C. Ball, and A.B. Nicotra. Compensation for herbivory by Cucumis sativus through increased photosynthetic capacity and efficiency. Oecologia, 134:167-175, 2003.

[23] J. H.M. Thornley and I. R. Johnson. Plant and Crop Modelling. A mathematical approach to plant and crop physiology. The Blackburn Press, USA, 1 edition, 1990.

[24] J.T. Trumble, D.M. Kolodny-Hirsh, and I.P. Ting. Plant compensatory for arthropod herbivory. Annual Reviews Entomology, 38:93-119, 1993.

[25] J. C. Van Lenteren. The state of commercial augmentative biological control: plenty of natural enemies, but a frustrating lack of uptake. BioControl, 57:1-20, 2012.

[26] J. Vos, J.B. Evers, G.H. Buck-Sorlin, B Andrieu, M. Chelle, and P.H.B de Visser. Functional-structural plant modelling : a new versatile tool in crop science. Journal of Experimental Botany, 61(8):2101, 2010.

[27] J. Vos, L.F.M. Marcelis, and J.B. Evers. Functional-Structural Plant Modelling in Crop Production, chapter 1 : Adding a dimension. Springer, 2007.

[28] A.G. Wheeler and E.R. Hoebeke. Coccinella novemnotata in northeastern nort america : historical occurence and current status (coleoptera : Coccinellidae). In Proceedings of the Entomological Society of Washington, volume 97, pages 701-716, 1995. 